OPEN ACCESS

Edited by: Peng Xia,

Life Sciences Institute Zhejiang

University, China

Reviewed by:

Nagaraj Balasubramanian, Indian Institute of Science Education and Research, Pune, India

*Correspondence:

Natalia A. Bulgakova

n.bulgakova@sheffield.ac.uk

Specialty section:

This article was submitted to Morphogenesis and Patterning, a section of the journal Frontiers in Cell and Developmental Biology

Received: 03 December 2021 Accepted: 31 December 2021 Published: 20 January 2022

Citation:

Ramírez Moreno M and Bulgakova NA (2022) The Cross-Talk Between EGFR and E-Cadherin.

Front. Cell Dev. Biol. 9:828673. doi: 10.3389/fcell.2021.828673

\section{The Cross-Talk Between EGFR and E-Cadherin}

\author{
Miguel Ramírez Moreno and Natalia A. Bulgakova* \\ School of Biosciences and Bateson Centre, The University of Sheffield, Sheffield, United Kingdom
}

Epidermal growth factor receptor (EGFR) and adhesion protein E-cadherin are major regulators of proliferation and differentiation in epithelial cells. Consistently, defects in both EGFR and E-cadherin-mediated intercellular adhesion are linked to various malignancies. These defects in either are further exacerbated by the reciprocal interactions between the two transmembrane proteins. On the one hand, EGFR can destabilize E-cadherin adhesion by increasing E-cadherin endocytosis, modifying its interactions with cytoskeleton and decreasing its expression, thus promoting tumorigenesis. On the other hand, E-cadherin regulates EGFR localization and tunes its activity. As a result, loss and mutations of E-cadherin promote cancer cell invasion due to uncontrolled activation of EGFR, which displays enhanced surface motility and changes in endocytosis. In this minireview, we discuss the molecular and cellular mechanisms of the cross-talk between E-cadherin and EGFR, highlighting emerging evidence for the role of endocytosis in this feedback, as well as its relevance to tissue morphogenesis, homeostasis and cancer progression.

Keywords: adhesion, morphogenesis, cancer, signalling, epithelia

\section{INTRODUCTION}

Few components are as determining for cell behaviour and fate as Epidermal Growth Factor Receptor (EGFR), which mediates such diverse processes as cell proliferation, survival, growth and differentiation (Wee and Wang, 2017). EGFR is a member of the ErbB family, which is respectively a part of the receptor tyrosine kinase superfamily (Herbst, 2004; Hynes and MacDonald, 2009). Downstream, it transduces multiple signalling pathways, most notably Ras/MAPK, PI3K/AKT/ mTOR and PLC/PKC signalling (Oda et al., 2005; Wee and Wang, 2017). Cancer cells often display upregulation of the EGFR signalling or receptor overexpression (Rowinsky, 2004; Guo et al., 2015; Wee and Wang, 2017; Sigismund et al., 2018). This highlights the importance of understanding the regulation and function of the EGFR signalling for novel cancer therapies (Yarden, 2001; Rowinsky, 2004; Vecchione et al., 2011; Sigismund et al., 2018).

Another important component controlling interactions between cells and with their environment is cell adhesion, mediated by Cell Adhesion Molecules (CAMs) (Gumbiner, 1996; Chothia and Jones, 1997). CAMs perform structural functions by linking extracellular space to the cystoskeleton inside cells (Parsons et al., 2010). However, rather than just gluing cells, adhesion also acts as a sensory tool to gather informational cues from the neighbouring cells and substrate (Geiger et al., 2009; Hamidi and Ivaska, 2021). Among the CAMs, E-cadherin is a the major component of the Adherens Junctions in epithelial cells, responsible for cell-cell adhesion (Takeichi, 1977; Chothia and Jones, 1997; Halbleib and Nelson, 2006).

Increasing evidence demonstrates interactions between the EGFR signalling and E-cadherinmediated cell-cell adhesion. An inverse correlation between levels of EGFR and E-cadherin was 


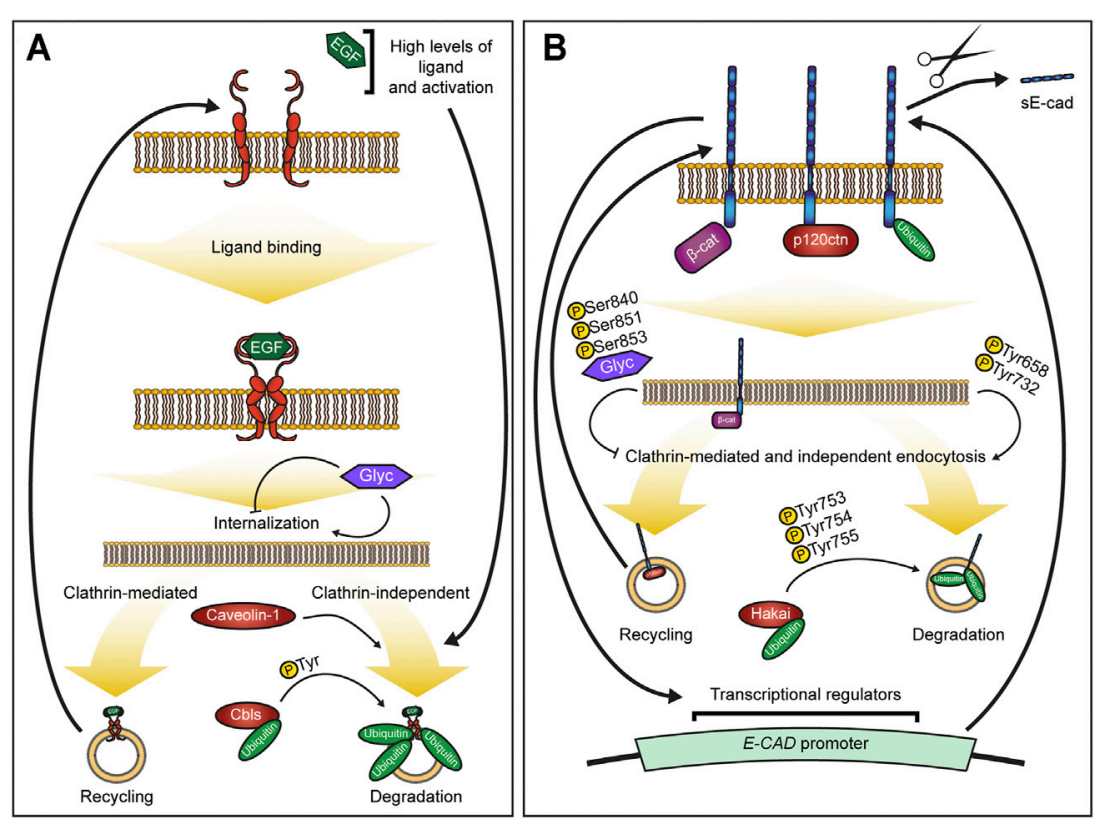

FIGURE 1 | Overview of mechanisms controlling EGFR and E-cadherin endocytosis. (A,B) - Summary of EGFR (A) and E-cadherin (B) regulation. Ligand-induced activation and dimerization of EGFR (A) trigger endocytosis of the receptor. The clathrin-mediated endocytosis is followed by recycling of the receptor and comprises most of the endocytic events, whereas clathrin-independent endocytosis, including caveolae, leads to EGFR degradation and is promoted upon a certain threshold of EGFR activation. Ubiquitination by Cbls ubiquitin ligases serves as a key cue for EGFR degradation and is modulated by EGFR phosphorylation. Levels of E-cadherin at the plasma membrane $\mathbf{( B )}$ are regulated by endocytosis, which is modulated by E-cadherin interactions with its binding partners. $\beta$-catenin ( $\beta$-cat) helps retain E-cadherin at the membrane, whereas p120-catenin (p120ctn) prevents E-cadherin endocytosis for degradation but promotes its recycling. Both clathrinmediated and independent pathways can be followed by either E-cadherin recycling or degradation, but the latter depends on E-cadherin ubiquitination by the ubiquitin ligase Hakai and potentially others. E-cadherin membrane presentation also regulates own gene (E-CAD) expression. Dynamics of both E-cadherin and EGFR is also regulated by glycosylation of their extracellular domains.

reported in various cancers including endometrial carcinoma, cholangiocarcinoma, head and neck squamous cell carcinoma, and breast carcinoma to name a few (Jones et al., 1996; Zuo et al., 2011; Clapéron et al., 2014; Yang et al., 2014). Here we summarize the existing data on these interactions and highlight the major remaining gaps.

\section{OVERVIEW OF EGFR REGULATION}

EGFR activity is highly dynamic with endocytosis playing a key role in controlling and fine-tuning EGFR signalling (Figure 1A). As it is reviewed elsewhere (see for example (Barbieri et al., 2016; Caldieri et al., 2018; Sigismund et al., 2018)), we will only briefly introduce the main aspects relevant to this minireview. EGFR can be endocytosed through both clathrin-mediated (CME) and independent (CIE) pathways, and the pathway choice is linked with the critical decision for EGFR: its recycling or degradation (Barbieri et al., 2016). The majority of activated EGFR appears to be internalized via $\mathrm{CME}$, which is followed by recycling thus prolonging the signalling (Huang et al., 2004; Sigismund et al., 2008; Rappoport and Simon, 2009; Chi et al., 2011). However, several CIE pathways also contribute to EGFR internalization, including caveolae - smooth vesicles formed by cholesterol- and sphingolipids-rich lipid rafts (Galbiati et al., 2001). This route was found to internalize EGFR at high ligand concentrations in HeLa but not HEp2 cells (Sigismund et al., 2005; Kazazic et al., 2006). Concurrently, lipid rafts and caveolae may prevent EGFR clustering and ligand-independent EGFR activation, which is observed upon cholesterol sequestration and caveolae inhibition with filipin III (Schnitzer et al., 1994; Lambert et al., 2006). Overall, CIE pathways appear to be activated at the high receptor or ligand concentrations and are followed by degradation (Sigismund et al., 2018, 2008). Such response makes physiological sense, promoting EGFR degradation as a countermeasure against hyperactivation (Barbieri et al., 2016). Therefore, it is not surprising that defects in EGFR degradation are seen, for example, in cholangiocarcinoma RBE and breast cancer cells (Gui et al., 2012; Pareja et al., 2012).

Intracellular trafficking of EGFR and its downstream targets are modulated by posttranslational modifications (Figure 1A). Ligand-activated EGFR undergoes dimerization and transautophosphorylation at several residues in the regulatory C-tail, as well as phosphorylation by kinases that act downstream (Miloso et al., 1995; Thelemann et al., 2005; Song et al., 2014; Wee and Wang, 2017). This attunes EGFR interactions, endocytosis and fate but also alters the cellular response to EGFR activation (Tong et al., 2009; Jones and Rappoport, 2014; Wee and Wang, 2017).

A core cue in determining the EGFR fate is ubiquitination, which is mostly placed by Cbl proteins (Levkowitz et al., 1999; Marmor and Yarden, 2004; Thien and Langdon, 2005; Huang 
et al., 2007). The ubiquitination depends on the present phosphotyrosines, highlighting feedbacks between receptor activation, endocytosis and posttranslational modifications (Sigismund et al., 2013). Deubiquitination of internalized EGFR promotes its recycling, bypassing the degradation pathway (Liu et al., 2013). A threshold EGFR activation is necessary for ubiquitination, switching from CME to CIE and subsequent degradation (Pinilla-Macua et al., 2017).

Finally, the EGFR extracellular domain is rich in sites whose $\mathrm{N}$-glycosylation affects EGFR signalling in multiple ways. Among other roles, N-glycosylation modifies EGFR folding thus regulating its ligand-binding; modulates endocytosis and intracellular trafficking of EGFR thus adjusting protein surface levels and signalling duration; prevents ligand-independent activation; and creates binding sites for extracellular lectins galectins -, which contribute to the assembly of supramolecular complexes and limit diffusion of receptors in the plasma membrane (recently reviewed in Porębska et al., 2021).

\section{OVERVIEW OF E-CADHERIN ADHESION REGULATION}

The membrane levels of E-cadherin determine adhesion strength, but also cell rearrangements and proliferation within the tissue (Chu et al., 2004; Ciesiolka et al., 2004; Mohan et al., 2018), whereas its loss is a hallmark of invasive carcinomas (Birchmeier and Behrens, 1994; Yu et al., 2019). The most characterized route to control E-cadherin surface levels is endocytosis (Figure 1B). Similar to EGFR, E-cadherin can be internalized by both CME and CIE (reviewed in (Nanes and Kowalczyk, 2012), which can be followed by its recycling or degradation (Le et al., 1999; Bulgakova et al., 2013; Cadwell et al., 2016; Brüser and Bogdan, 2017). The fate of internalized E-cadherin is not ultimately linked to the internalization pathway; CME can be followed by either degradation or recycling (Le et al., 1999; Xiao et al., 2003). Instead, the p120-catenin protein, which directly binds the E-cadherin intracellular domain, might be determining the outcome; while p120-catenin binding prevents E-cadherin CME followed by degradation, it also recruits Numb to promote CME followed by recycling (Ishiyama et al., 2010; Sato et al., 2011).

Posttranslational modifications modulate E-cadherin stability, affinity to binding partners and trafficking (Figueiredo et al., 2013; Brüser and Bogdan, 2017). Phosphorylation at Ser840, Ser851 and Ser853 increases E-cadherin affinity to $\beta$-catenin and stabilizes adhesion by preventing E-cadherin endocytosis and degradation (Lickert et al., 2000; Jaggi et al., 2005; McEwen et al., 2014). In contrast, phosphorylation of Tyr658 and Tyr732 of VE-cadherin reduces its binding to $\beta$-catenin and p120catenin, negatively affecting its function (Jeanes et al., 2008; Bertocchi et al., 2012; Chen et al., 2016). Phosphorylation of E-cadherin at Tyr753-755 creates a docking site for the E3 ubiquitin ligase Hakai, and possibly others such as March8 (Fujita et al., 2002; Pece and Gutkind, 2002; Kaido et al., 2009; Kim et al., 2014, p. 8). Hakai promotes E-cadherin degradation and competes with p120-catenin for E-cadherin binding
(Hartsock and Nelson, 2012). Moreover, Hakai alongside Src also stabilizes $\delta$-catenin, which promotes E-cadherin processing (Palacios et al., 2005; Kim et al., 2012; Shrestha et al., 2017). Various proteinases including matrix metalloproteinase-2 (MMP-2) and matrix metalloproteinase-9 (MMP-9) - whose high levels correlate metastasis and poor prognosis of multiple cancers - can induce proteolytic cleavage of E-cadherin extracellular domain (Roomi et al., 2009; Li et al., 2017). Upon the cleavage, the extracellular proteolytic fragment (soluble E-cadherin, sE-cad) is released into extracellular space, where it has multiple effects including interfering with E-cadherin adhesion, signalling activities and antitumor immune response (Hu et al., 2016). Additionally, glycosylation of the E-cadherin extracellular domain modulates E-cadherin adhesive function and endocytic turnover (Zhao et al., 2008; Advedissian et al., 2017).

Finally, the regulation of E-cadherin transcription involves a complex network of transcriptional repressors, activators, and epigenetic modifiers (Ramirez Moreno et al., 2021). Among others, the closely related transcriptional repressors SLUG and SNAIL (also known as SNAI2 and SNAI1) directly repress E-cadherin transcription by binding conserved E-boxes in its promoter (Batlle et al., 2000; Cano et al., 2000; Bolós et al., 2003). Consistently, changes in the machinery that modulates its expression often lead to loss of E-cadherin in cancers (Bringuier et al., 1999; Bruner and Derksen, 2018; Ramirez Moreno et al., 2021).

\section{REGULATION OF E-CADHERIN BY EGFR SIGNALLING}

Changes to EGFR signalling promote epithelial-to-mesenchymal transition (EMT), at least in part by downregulating E-cadherin. EGFR is overexpressed in $70 \%$ of malignant ovarian tumours and $85 \%$ of salivary adenoid cystic carcinomas, leading to increased mRNA levels of SLUG (Bartlett et al., 1996; Cheng et al., 2012, 2013; Wang et al., 2018). In ovarian cancer cells, EGFR activation promotes SLUG transcription by inducing the expression of the transcription factor Egr-1, which directly binds to the SLUG promoter (Cheng et al., 2013). The relevance of elevated SLUG expression remains controversial: while inhibiting SLUG expression in ovarian SKOV3 and OVACR5 and oviductal OE-E6/E7 cells restored E-cadherin expression and limited cell invasiveness, silencing SLUG did not inhibit EMT in salivary adenoid cystic carcinoma cells (Cheng et al., 2012, 2013; Wang et al., 2018). In ovarian cancer cells SKOV3 and OVCAR3, EGFR activation also increased SNAIL mRNA levels, which required EGF-induced $\mathrm{H}_{2} \mathrm{O}_{2}$ production and p38 MAPK activation (Cheng et al., 2010). In contrast, in salivary adenoid cystic carcinoma cells, EGF-induced EGFR activation lincreases levels of SNAIL protein without altering its mRNA levels (Cheng et al., 2012, 2013; Wang et al., 2018). In both cases, however, silencing SNAIL reduced EMT and invasiveness (Cheng et al., 2010; Wang et al., 2018). Curiously, in oviductal epithelial cells, EGFR activation alters neither mRNA nor protein levels of SNAIL (Cheng et al., 2012). 


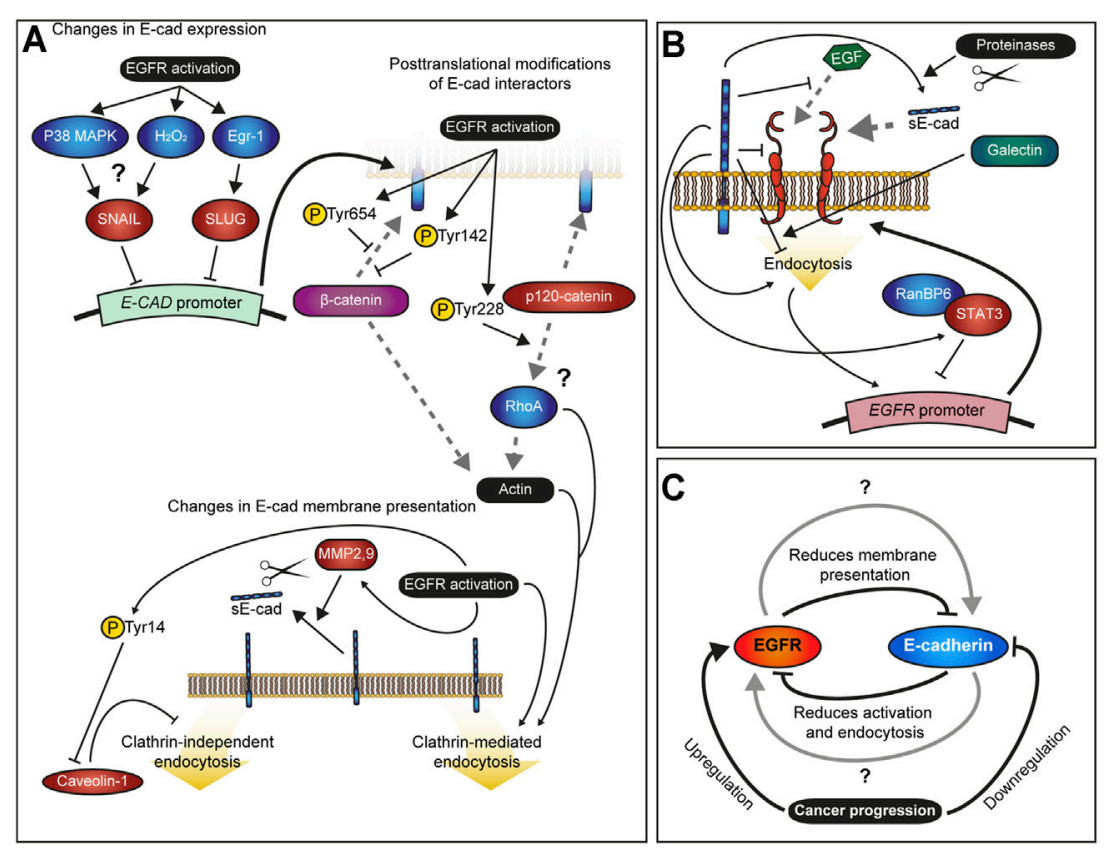

FIGURE 2 | Interactions between EGFR and E-cadherin. (A) - Summary of the known effects of EGFR activation on E-cadherin. EGFR signalling downregulates Ecadherin (E-CAD) gene expression via the transcriptional repressors SNAIL and SLUG. By promoting phosphorylation, it also destabilizes membrane E-cadherin by reducing its affinity with $\beta$-catenin and the subsequent connection to the actin cytoskeleton as well as alters interactions between p120-catenin with RhoA. Additionally, EGFR signalling increases E-cadherin endocytosis by blocking Caveolin-1 activity, a negative regulator of the EGFR pathway itself, and promotes processing of E-cadherin into soluble E-cadherin (sE-cad) through activation of the Matrix Metalloproteinases (MMPs) 2 and 9. Grey dashed lines indicate protein binding. (B) Summary of the known effects of E-cadherin on EGFR. E-cadherin stabilizes EGFR at the membrane, blocks its activation by EGF and reduces its internalization. Through STAT3 and RanBP6, E-cadherin represses EGFR gene expression. Additionally, sE-Cad is an agonistic ligand of EGFR, and therefore E-cadherin cleavage, which is promoted by EGFR activity, positively regulates EGFR signalling. (C) - Model of the feedback mechanism between EGFR activity and E-cadherin; the negative feedback loop between the two leads to stable EGFR activation and loss of E-cadherin in cancer cells. Gray arrows indicate unknown mechanisms by which both transmembrane proteins coexist and fine-tune each other in normal tissue during development and homeostasis.

EGFR activation also downregulates E-cadherin through several posttranscriptional mechanisms (Figure 2A). Active EGFR induces phosphorylation of both $\beta$ - and p120-catenin (Hoschuetzky et al., 1994; Hazan and Norton, 1998; Mariner et al., 2004). Phosphorylation of $\beta$-catenin at Tyr654 and Tyr142 reduces its affinity for E-cadherin and $\alpha$-catenin binding, respectively (Roura et al., 1999; Piedra et al., 2003), which could be responsible for the dissociation of E-cadherin from actin cytoskeleton following EGF treatment in breast cancer cells MDA-MB-468 (Hazan and Norton, 1998). EGFR-mediated weakening of association between E-cadherin and actin cytoskeleton may contribute to normal development by enabling cell rearrangements through remodelling of cell contacts, but also promote EMT in malignancy through fragmentation of adherens junctions and cortical actin bundle (Zhitnyak et al., 2020; Fu et al., 2021).

Similarly, EGFR promotes tyrosine phosphorylation of p120catenin at Tyr228 (Mariner et al., 2004), although the exact intermediate of this phosphorylation is unclear (Alemà and Salvatore, 2007). This residue is present in both common isoforms of p120catenin - mesenchymal isoform 1 and epithelial isoform 3 (Reynolds and Roczniak-Ferguson, 2004). While Tyr228 phosphorylation does not affect p120-catenin binding to E-cadherin and its endocytosis directly, it increases p120-catenin affinity for RhoA binding (Mariner et al., 2004; Castaño et al., 2007; Kourtidis et al., 2013). The effect of this phosphorylation appears to be context-dependent. In E-cadherin-deficient breast cancer cells MDA-MB-231, the binding of p120-catenin N-terminus inhibits RhoA activity (Yanagisawa et al., 2008). Conversely, deletion of the p120catenin N-terminus inhibits EGF-induced motility, whereas ectopic expression of full-length p120-catenin promotes cell motility in keratinocytes through activation of RhoA and cytoskeletal rearrangements (Cozzolino et al., 2003). This discrepancy in effects of p120-catenin N-terminus on RhoA activity and cell behaviour is consistent with the differences in levels and roles of Tyr228 phosphorylation of p120-catenin in cancer cells. In colon adenocarcinoma cells, phosphorylation of Tyr228 correlates with better prognosis and inhibits cell invasion (Ding et al., 2019), whereas in the breast cancer cells MDA-MB231 it is essential for the invasiveness-promoting activity of p120catenin isoform 1 (Yanagisawa et al., 2008; Kourtidis et al., 2015). In either case, the changes in RhoA activity are likely to alter (promote or inhibit) E-cadherin endocytosis depending on the context (Lee and Harris, 2013; Kourtidis et al., 2015; Greig and Bulgakova, 2020).

EGFR activation promotes E-cadherin endocytosis through various routes. In MCF-7 cells, stimulation with EGF promotes 
either macropinocytosis of E-cadherin or its endocytosis mediated by the small GTPase Arf6, which is likely to be clathrin-dependent (Bryant et al., 2007; Kon et al., 2008). Here, the internalization route might depend on the levels of E-cadherin expression. EGFR activation by EGF also leads to E-cadherin internalization in A431 epidermoid carcinoma cells and A549 lung cancer cells via caveolae (Lu et al., 2003). EGFR activation leads to caveolin-1 phosphorylation at Tyr14 and its fast redistribution from the plasma membrane (Pol et al., 2000; Orlichenko et al., 2006). Caveolin-1 negatively regulates the caveolae-mediated endocytosis due to its ability to stabilize caveolae association with the plasma membrane (Le et al., 2002; Simón et al., 2020). Consistently, disruption of caveolae using filipin III blocks E-cadherin endocytosis following EGFR activation (Lu et al., 2003). In addition, chronic EGFR activity inhibits mRNA expression of caveolin-1 (Lu et al., 2003), which is likely to ensure sustained caveolae endocytosis of surface E-cadherin. Curiously, knockdown on caveolin-1 is sufficient to downregulate E-cadherin but also leads to SNAIL overexpression (Lu et al., 2003). While EGF-activated EGFR is not internalized by caveolae (Kazazic et al., 2006), disruption of lipid rafts leads to ligand-independent EGFR activation (Lambert et al., 2006). Therefore, we speculate that the changes in gene expression of SNAIL and E-cadherin following caveolin-1 knockdown might be due to an indirect effect of ligandindependent EGFR activation.

Finally, EGFR activation may further inhibit E-cadherinmediated adhesion through proteolytic cleavage of E-cadherin. The secretion of matrix metalloproteinase-2 (MMP-2) is enhanced by EGF supplementation in salivary gland pleomorphic adenoma cells (Navarini et al., 2017), whereas in some ovarian cancer cell lines (OVEA6 and OVCA 429 but not DOV13 and OVCA 432) EGFR activation increases the expression of matrix metalloproteinase-9 (MMP-9) (Ellerbroek et al., 1998). Conversely, EGF produced by lymphoma cells inhibits MMP-9 expression in neighbouring stromal cells through induction of Egr-1 expression (Bouchard et al., 2010). Such context-dependency indicates that these effects might be indirect and rely on additional factors present in each case.

To summarize, at least five molecular routes links EGFR activity and E-cadherin (Figure 2A). Altogether, this ensures robust inhibition of E-cadherin-mediated adhesion, promoting EMT and cell migration in cancer.

\section{REGULATION OF EGFR SIGNALLING BY E-CADHERIN}

The extracellular domain of E-cadherin directly binds EGFR in both mammalian and fly cells (Dumstrei et al., 2002; Qian et al., 2004). This binding promotes EGFR localization at the sites of E-cadherin-mediated adhesion, but also interferes with EGF binding to EGFR and reduces the mobility of EGFR in the plasma membrane (Figure 2B) (Qian et al., 2004; Rübsam et al., 2017). Consequently, the loss of E-cadherin leads to increased ligand binding to EGFR, but at the same time promotes EGFR mobility which may stimulate EGFR dimerization and further boost its activation (Bremm et al., 2008). As the result, the loss of E-cadherin often observed in cancer cells leads to activation of EGFR signalling, thus, promoting cancer cell dissemination (Takahashi and Suzuki, 1996; Bae et al., 2013). Conversely, in some contexts, E-cadherin may have an opposite effect as the induction of E-cadherin adhesion assembly in HaCat keratinocyte cells and MCF-10A mammary epithelial cells leads to EGF-independent EGFR activation and requires the extracellular domain of E-cadherin (Pece and Gutkind, 2000; Fedor-Chaiken et al., 2003). Besides EGF, EGFR can be activated by other ligands (Harris et al., 2003; Singh et al., 2016), including sE-cad (Brouxhon et al., 2014; Hu et al., 2016). Moreover, in MCF7 and MDA-MB-231 breast cancer cell lines sE-cad shows a stronger effect than EGF, and acts additively with it (Brouxhon et al., 2014).

One of the possible, though unexplored, explanations for the observed opposite effects of E-cadherin on EGFR, is its potential effect on EGFR endocytosis (Figure 2B). As described above, endocytosis of EGFR is a powerful mechanism of tuning its activity. Indeed, increased activation of EGFR in cells expressing the E-cadherin mutant, which lacks the exon 8 in its extracellular domain (corresponding to the E-cadherin ectodomain 2) but still binds EGFR, is accompanied by the decreased internalization of EGFR from the plasma membrane upon EGF stimulation (Bremm et al., 2008), indicating that the ectodomain 2 promotes EGFR endocytosis. In contrast, the ectodomain 3 is connected to EGFR by galectin-7, which negatively regulates EGFR endocytosis (Proux-Gillardeaux et al., 2021). Thus, E-cadherin extracellular domain may promote or inhibit EGFR endocytosis depending on the context.

In addition to regulation of EGFR activity at the cell surface, E-cadherin downregulation leads to EGFR upregulation on mRNA level in cells from squamous cell carcinoma of the head and neck (Wang et al., 2011). This upregulation might be an indirect effect of positive feedback whereby EGFR activation at the plasma membrane results in increased expression of the EGFR gene (Clark et al., 1985; Oldrini et al., 2017). This feedback was suggested to act to restore levels of EGFR following its activation, internalization and consequent degradation, therefore ensuring the robustness of EGFR signalling (Oldrini et al., 2017). The feedback from EGFR to its own gene expression involves the signal transducer and activator of transcription 3 (STAT3) protein. STAT3 binds EGFR promoter and inhibits its transcription in RanBP6dependent manner in HEK-293T human kidney cells. When demand arises this inhibition can be lifted, for example when additional production of EGFR is required following its ligandinduced degradation (Oldrini et al., 2017). At the same time, inhibition of STAT3 phosphorylation is sufficient to increase levels of EGFR mRNA (Oldrini et al., 2017), whereas E-cadherin promotes STAT3 activation in mouse embryonic stem cells (del Valle et al., 2013), suggesting that it can contribute to this feedback.

Therefore, E-cadherin in most cases inhibits EGFR through a combination of modulating its behaviour at the cell surface and promoting transcriptional silencing (Figure 2B). However, 
E-cadherin acts in more than one way and in some contexts, may activate EGFR instead.

\section{CONCLUSION}

Both EGFR and E-cadherin are vital for normal development, highly dynamic and often dysregulated in cancer cells. In the latter, there is feedback between the two proteins; EGFR downregulates E-cadherin through multiple mechanisms and vice versa (Figures 2A,B). Such feedback should lead to fast amplification of adhesion loss and EGFR activation, promoting invasiveness and proliferation of a tumour (Figure 2C). However, if the interaction between the two proteins were limited to this feedback, it would be impossible for simultaneous E-cadherin-mediated adhesion and EGFR signalling in a cell. Meanwhile, multiple examples of such cells exist. Human skin stem cells require EGFR activity for proliferation and express E-cadherin, even if at lower levels than other keratinocytes (Molès and Watt, 1997; Brechbuhl et al., 2014). Similarly, during Drosophila wing development EGFR activity is required for specification of veins and leads to a basal shift in E-cadherin localization

\section{REFERENCES}

Advedissian, T., Proux-Gillardeaux, V., Nkosi, R., Peyret, G., Nguyen, T., Poirier, F., et al. (2017). E-cadherin Dynamics Is Regulated by Galectin-7 at Epithelial Cell Surface. Sci. Rep. 7, 17086. doi:10.1038/s41598-017-17332-y

Alemà, S., and Salvatore, A. M. (2007). p120 Catenin and Phosphorylation: Mechanisms and Traits of an Unresolved Issue. Biochim. Biophys. Acta (Bba) - Mol. Cel Res. 1773, 47-58. doi:10.1016/j.bbamcr.2006.06.001

Bae, G.-Y., Choi, S.-J., Lee, J.-S., Jo, J., Lee, J., Kim, J., et al. (2013). Loss of E-Cadherin Activates EGFR-MEK/ERK Signaling, Which Promotes Invasion via the ZEB1/MMP2 axis in Non-small Cell Lung Cancer. Oncotarget 4, 2512-2522. doi:10.18632/oncotarget.1463

Barbieri, E., Di Fiore, P. P., and Sigismund, S. (2016). Endocytic Control of Signaling at the Plasma Membrane. Curr. Opin. Cel Biol. 39, 21-27. doi:10.1016/j.ceb.2016.01.012

Bartlett, J., Langdon, S., Simpson, B., Stewart, M., Katsaros, D., Sismondi, P., et al. (1996). The Prognostic Value of Epidermal Growth Factor Receptor mRNA Expression in Primary Ovarian Cancer. Br. J. Cancer 73, 301-306. doi:10.1038/ bjc. 1996.53

Batlle, E., Sancho, E., Francí, C., Domínguez, D., Monfar, M., Baulida, J., et al. (2000). The Transcription Factor Snail Is a Repressor of E-Cadherin Gene Expression in Epithelial Tumour Cells. Nat. Cel Biol 2, 84-89. doi:10.1038/35000034

Bertocchi, C., Vaman Rao, M., and Zaidel-Bar, R. (2012). Regulation of Adherens junction Dynamics by Phosphorylation Switches. J. Signal Transduction 2012, 1-14. doi:10.1155/2012/125295

Birchmeier, W., and Behrens, J. (1994). Cadherin Expression in Carcinomas: Role in the Formation of Cell Junctions and the Prevention of Invasiveness. Biochim. Biophys. Acta (Bba) - Rev. Cancer 1198, 11-26. doi:10.1016/0304-419x(94) 90003-5

Bolo's, V., Peinado, H., Pe'rez-Moreno, M. A., Fraga, M. F., Esteller, M., and Cano, A. (2003). The Transcription Factor Slug Represses E-Cadherin Expression and Induces Epithelial to Mesenchymal Transitions: a Comparison with Snail and E47 Repressors. J. Cel Sci. 116, 499-511. doi:10.1242/jcs.00224

Bouchard, F., Bélanger, S. D., Biron-Pain, K., and St-Pierre, Y. (2010). EGR-1 Activation by EGF Inhibits MMP-9 Expression and Lymphoma Growth. Blood 116, 759-766. doi:10.1182/blood-2009-12-257030 without adhesion loss (O'Keefe et al., 2007). We speculate that expression levels and endocytic trafficking of both proteins play an important role in their effects on each other, as well as the mechanical environment of the cells. Thus, upon mechanical stress, EGFR promotes E-cadherinmediated cell stiffening through activation of the Abl kinase, leading to the recruitment of vinculin to the adhesion sites (Sehgal et al., 2018). Discovering molecular mechanisms of how EGFR activity and E-cadherin-mediated adhesion coexist in normal tissues is essential for understanding the causes of the amplifying feedback between them in cancer cells and developing approaches to break this feedback.

\section{AUTHOR CONTRIBUTIONS}

$\mathrm{MM}$ and NB wrote the manuscript.

\section{FUNDING}

This work was supported by a grant from the UKRI BBSRC (BB/ P007503/1) to NB.

Brechbuhl, H. M., Li, B., Smith, R. W., and Reynolds, S. D. (2014). Epidermal Growth Factor Receptor Activity Is Necessary for Mouse Basal Cell Proliferation. Am. J. Physiology-Lung Cell Mol. Physiol. 307, L800-L810. doi:10.1152/ajplung.00201.2014

Bremm, A., Walch, A., Fuchs, M., Mages, J., Duyster, J., Keller, G., et al. (2008). Enhanced Activation of Epidermal Growth Factor Receptor Caused by TumorDerived E-Cadherin Mutations. Cancer Res. 68, 707-714. doi:10.1158/00085472.CAN-07-1588

Bringuier, P.-P., Giroldi, L. A., Umbas, R., Shimazui, T., and Schalken, J. A. (1999). Mechanisms Associated with Abnormal E-Cadherin Immunoreactivity in Human Bladder Tumors. Int. J. Cancer 83, 591-595. doi:10.1002/(sici)10970215(19991126)83:5<591:aid-ijc3>3.0.co;2-6

Brouxhon, S. M., Kyrkanides, S., Teng, X., O’Banion, M. K., Clarke, R., Byers, S., et al. (2014). Soluble-E-cadherin Activates HER and IAP Family Members in HER2+ and TNBC Human Breast Cancers. Mol. Carcinog. 53, 893-906. doi: $10.1002 / \mathrm{mc} .22048$

Bruner, H. C., and Derksen, P. W. B. (2018). Loss of E-cadherin-dependent CellCell Adhesion and the Development and Progression of Cancer. Cold Spring Harb Perspect. Biol. 10, a029330. doi:10.1101/cshperspect.a029330

Brüser, L., and Bogdan, S. (2017). Adherens Junctions on the Move-Membrane Trafficking of E-Cadherin. Cold Spring Harb Perspect. Biol. 9, a029140. doi:10.1101/cshperspect.a029140

Bryant, D. M., Kerr, M. C., Hammond, L. A., Joseph, S. R., Mostov, K. E., Teasdale, R. D., et al. (2007). EGF Induces Macropinocytosis and SNX1-Modulated Recycling of E-Cadherin. J. Cel Sci. 120, 1818-1828. doi:10.1242/jcs.000653

Bulgakova, N. A., Grigoriev, I., Yap, A. S., Akhmanova, A., and Brown, N. H. (2013). Dynamic Microtubules Produce an Asymmetric E-Cadherin-Bazooka Complex to Maintain Segment Boundaries. J. Cel Biol 201, 887-901. doi:10.1083/jcb.201211159

Cadwell, C. M., Su, W., and Kowalczyk, A. P. (2016). Cadherin Tales: Regulation of Cadherin Function by Endocytic Membrane Trafficking. Traffic 17, 1262-1271. doi: $10.1111 /$ tra. 12448

Caldieri, G., Malabarba, M. G., Di Fiore, P. P., and Sigismund, S. (2018). EGFR Trafficking in Physiology and Cancer. Prog. Mol. Subcell Biol. 57, 235-272. doi:10.1007/978-3-319-96704-2_9

Cano, A., Pérez-Moreno, M. A., Rodrigo, I., Locascio, A., Blanco, M. J., del Barrio, M. G., et al. (2000). The Transcription Factor Snail Controls Epithelial- 
Mesenchymal Transitions by Repressing E-Cadherin Expression. Nat. Cel Biol 2, 76-83. doi:10.1038/35000025

Castaño, J., Solanas, G., Casagolda, D., Raurell, I., Villagrasa, P., Bustelo, X. R., et al. (2007). Specific Phosphorylation of P120-Catenin Regulatory Domain Differently Modulates its Binding to RhoA. Mol. Cel Biol 27, 1745-1757. doi:10.1128/MCB.01974-06

Chen, C.-L., Wang, S.-H., Chan, P.-C., Shen, M.-R., and Chen, H.-C. (2016). Phosphorylation of E-Cadherin at Threonine 790 by Protein Kinase C $\delta$ Reduces $\beta$-catenin Binding and Suppresses the Function of E-Cadherin. Oncotarget 7, 37260-37276. doi:10.18632/oncotarget.9403

Cheng, J.-C., Chang, H.-M., and Leung, P. C. K. (2013). Egr-1 Mediates Epidermal Growth Factor-Induced Downregulation of E-Cadherin Expression via Slug in Human Ovarian Cancer Cells. Oncogene 32, 1041-1049. doi:10.1038/ onc.2012.127

Cheng, J.-C., Chang, H.-M., and Leung, P. C. K. (2012). Epidermal Growth Factor Induces Human Oviductal Epithelial Cell Invasion by Down-Regulating E-Cadherin Expression. J. Clin. Endocrinol. Metab. 97, E1380-E1389. doi:10.1210/jc.2011-2751

Cheng, J.-C., Klausen, C., and Leung, P. C. K. (2010). Hydrogen Peroxide Mediates EGF-Induced Down-Regulation of E-Cadherin Expression via P38 MAPK and Snail in Human Ovarian Cancer Cells. Mol. Endocrinol. 24, 1569-1580. doi:10.1210/me.2010-0034

Chi, S., Cao, H., Wang, Y., and McNiven, M. A. (2011). Recycling of the Epidermal Growth Factor Receptor Is Mediated by a Novel Form of the Clathrin Adaptor Protein Eps15. J. Biol. Chem. 286, 35196-35208. doi:10.1074/jbc.M111.247577

Chothia, C., and Jones, E. Y. (1997). The Molecular Structure of Cell Adhesion Molecules. Annu. Rev. Biochem. 66, 823-862. doi:10.1146/ annurev.biochem.66.1.823

Chu, Y.-S., Thomas, W. A., Eder, O., Pincet, F., Perez, E., Thiery, J. P., et al. (2004). Force Measurements in E-Cadherin-Mediated Cell Doublets Reveal Rapid Adhesion Strengthened by Actin Cytoskeleton Remodeling through Rac and Cdc42. J. Cel Biol. 167, 1183-1194. doi:10.1083/jcb.200403043

Ciesiolka, M., Delvaeye, M., Van Imschoot, G., Verschuere, V., McCrea, P., van Roy, F., et al. (2004). p120 Catenin Is Required for Morphogenetic Movements Involved in the Formation of the Eyes and the Craniofacial Skeleton in Xenopus. J. Cel Sci. 117, 4325-4339. doi:10.1242/jcs.01298

Clapéron, A., Mergey, M., Nguyen Ho-Bouldoires, T. H., Vignjevic, D., Wendum, D., Chrétien, Y., et al. (2014). EGF/EGFR axis Contributes to the Progression of Cholangiocarcinoma through the Induction of an Epithelial-Mesenchymal Transition. J. Hepatol. 61, 325-332. doi:10.1016/j.jhep.2014.03.033

Clark, A. J., Ishii, S., Richert, N., Merlino, G. T., and Pastan, I. (1985). Epidermal Growth Factor Regulates the Expression of its Own Receptor. Proc. Natl. Acad. Sci. 82, 8374-8378. doi:10.1073/pnas.82.24.8374

Cozzolino, M., Stagni, V., Spinardi, L., Campioni, N., Fiorentini, C., Salvati, E., et al. (2003). p120 Catenin Is Required for Growth Factor-dependent Cell Motility and Scattering in Epithelial Cells. MBoC 14, 1964-1977. doi:10.1091/mbc.e0208-0469

del Valle, I., Rudloff, S., Carles, A., Li, Y., Liszewska, E., Vogt, R., et al. (2013). E-cadherin Is Required for the Proper Activation of the Lifr/Gp130 Signaling Pathway in Mouse Embryonic Stem Cells. Development 140, 1684-1692. doi:10.1242/dev.088690

Ding, X., Wang, X., Lu, S., Gao, X., and Ju, S. (2019). P120-Catenin and its Phosphorylation on Tyr228 Inhibits Proliferation and Invasion in Colon Adenocarcinoma Cells. Ott 12, 10213-10225. doi:10.2147/OTT.S211973

Dumstrei, K., Wang, F., Shy, D., Tepass, U., and Hartenstein, V. (2002). Interaction between EGFR Signaling and DE-cadherin during Nervous System Morphogenesis. Development 129, 3983-3994. doi:10.1242/dev.129.17.3983

Ellerbroek, S. M., Hudson, L. G., and Stack, M. S. (1998). Proteinase Requirements of Epidermal Growth Factor-Induced Ovarian Cancer Cell Invasion. Int. J. Cancer 78, 331-337. doi:10.1002/(SICI)1097-0215(19981029)78:3<331: AID-IJC13>3.0.CO;2-9

Fedor-Chaiken, M., Hein, P. W., Stewart, J. C., Brackenbury, R., and Kinch, M. S. (2003). E-cadherin Binding Modulates EGF Receptor Activation. Cel Commun. Adhes. 10, 105-118. doi:10.1080/cac.10.2.105.118

Figueiredo, J., Söderberg, O., Simões-Correia, J., Grannas, K., Suriano, G., and Seruca, R. (2013). The Importance of E-Cadherin Binding Partners to Evaluate the Pathogenicity of E-Cadherin Missense Mutations Associated to HDGC. Eur. J. Hum. Genet. 21, 301-309. doi:10.1038/ejhg.2012.159
Fu, C., Arora, A., Engl, W., Sheetz, M., and Viasnoff, V. (2021). Cooperative Regulation of Adherens junction Elongation through Epidermal Growth Factor Receptor (EGFR) Activation. doi:10.1101/2021.05.25.445561

Fujita, Y., Krause, G., Scheffner, M., Zechner, D., Leddy, H. E. M., Behrens, J., et al. (2002). Hakai, a C-cbl-like Protein, Ubiquitinates and Induces Endocytosis of the E-Cadherin Complex. Nat. Cel Biol 4, 222-231. doi:10.1038/ncb758

Galbiati, F., Razani, B., and Lisanti, M. P. (2001). Emerging Themes in Lipid Rafts and Caveolae. Cell 106, 403-411. doi:10.1016/S0092-8674(01)00472-X

Geiger, B., Spatz, J. P., and Bershadsky, A. D. (2009). Environmental Sensing through Focal Adhesions. Nat. Rev. Mol. Cel Biol 10, 21-33. doi:10.1038/ nrm2593

Greig, J., and Bulgakova, N. A. (2020). Interplay between Actomyosin and E-Cadherin Dynamics Regulates Cell Shape in the Drosophila Embryonic Epidermis. J. Cel Sci 133. doi:10.1242/jcs.242321

Gui, A., Kobayashi, A., Motoyama, H., Kitazawa, M., Takeoka, M., and Miyagawa, S. (2012). Impaired Degradation Followed by Enhanced Recycling of Epidermal Growth Factor Receptor Caused by Hypo-Phosphorylation of Tyrosine 1045 in RBE Cells. BMC Cancer 12, 179. doi:10.1186/1471-2407-12-179

Gumbiner, B. M. (1996). Cell Adhesion: the Molecular Basis of Tissue Architecture and Morphogenesis. Cell 84, 345-357. doi:10.1016/s0092-8674(00)81279-9

Guo, G., Gong, K., Wohlfeld, B., Hatanpaa, K. J., Zhao, D., and Habib, A. A. (2015). Ligand-independent EGFR Signaling. Cancer Res. 75, 3436-3441. doi:10.1158/ 0008-5472.CAN-15-0989

Halbleib, J. M., and Nelson, W. J. (2006). Cadherins in Development: Cell Adhesion, Sorting, and Tissue Morphogenesis. Genes Dev. 20, 3199-3214. doi:10.1101/gad.1486806

Hamidi, H., and Ivaska, J. (2021). Food for Thought: How Cell Adhesion Coordinates Nutrient Sensing. J. Cel Biol. 220. doi:10.1083/jcb.202103128

Harris, R., Chung, E., and Coffey, R. J. (2003). EGF Receptor Ligands. Exp. Cel Res. 284, 2-13. doi:10.1016/s0014-4827(02)00105-2

Hartsock, A., and Nelson, W. J. (2012). Competitive Regulation of E-Cadherin Juxtamembrane Domain Degradation by P120-Catenin Binding and HakaiMediated Ubiquitination. PLoS One 7, e37476. doi:10.1371/ journal.pone.0037476

Hazan, R. B., and Norton, L. (1998). The Epidermal Growth Factor Receptor Modulates the Interaction of E-Cadherin with the Actin Cytoskeleton. J. Biol. Chem. 273, 9078-9084. doi:10.1074/jbc.273.15.9078

Herbst, R. S. (2004). Review of Epidermal Growth Factor Receptor Biology. Int. J. Radiat. Oncolog ${ }^{*}$ Biology ${ }^{*}$ Physics 59, S21-S26. doi:10.1016/ j.ijrobp.2003.11.041

Hoschuetzky, H., Aberle, H., and Kemler, R. (1994). Beta-catenin Mediates the Interaction of the Cadherin-Catenin Complex with Epidermal Growth Factor Receptor. J. Cel Biol 127, 1375-1380. doi:10.1083/jcb.127.5.1375

Hu, Q.-P., Kuang, J.-Y., Yang, Q.-K., Bian, X.-W., and Yu, S.-C. (2016). Beyond a Tumor Suppressor: Soluble E-Cadherin Promotes the Progression of Cancer. Int. J. Cancer 138, 2804-2812. doi:10.1002/ijc.29982

Huang, F., Goh, L. K., and Sorkin, A. (2007). EGF Receptor Ubiquitination Is Not Necessary for its Internalization. Proc. Natl. Acad. Sci. 104, 16904-16909. doi:10.1073/pnas.0707416104

Huang, F., Khvorova, A., Marshall, W., and Sorkin, A. (2004). Analysis of ClathrinMediated Endocytosis of Epidermal Growth Factor Receptor by RNA Interference. J. Biol. Chem. 279, 16657-16661. doi:10.1074/jbc.C400046200

Hynes, N. E., and MacDonald, G. (2009). ErbB Receptors and Signaling Pathways in Cancer. Curr. Opin. Cel Biol. 21, 177-184. doi:10.1016/j.ceb.2008.12.010

Ishiyama, N., Lee, S.-H., Liu, S., Li, G.-Y., Smith, M. J., Reichardt, L. F., et al. (2010). Dynamic and Static Interactions between P120 Catenin and E-Cadherin Regulate the Stability of Cell-Cell Adhesion. Cell 141, 117-128. doi:10.1016/ j.cell.2010.01.017

Jaggi, M., Rao, P. S., Smith, D. J., Wheelock, M. J., Johnson, K. R., Hemstreet, G. P., et al. (2005). E-cadherin Phosphorylation by Protein Kinase D1/protein Kinase $\mathrm{C}\{\mathrm{mu}\}$ Is Associated with Altered Cellular Aggregation and Motility in Prostate Cancer. Cancer Res. 65, 483-492.

Jeanes, A., Gottardi, C. J., and Yap, A. S. (2008). Cadherins and Cancer: How Does Cadherin Dysfunction Promote Tumor Progression? Oncogene 27, 6920-6929. doi:10.1038/onc.2008.343

Jones, J., Royall, J., and Walker, R. (1996). E-cadherin Relates to EGFR Expression and Lymph Node Metastasis in Primary Breast Carcinoma. Br. J. Cancer 74, 1237-1241. doi:10.1038/bjc.1996.522 
Jones, S., and Rappoport, J. Z. (2014). Interdependent Epidermal Growth Factor Receptor Signalling and Trafficking. Int. J. Biochem. Cel Biol. 51, 23-28. doi:10.1016/j.biocel.2014.03.014

Kaido, M., Wada, H., Shindo, M., and Hayashi, S. (2009). Essential Requirement for RING finger E3 Ubiquitin Ligase Hakai in Early Embryonic Development ofDrosophila. Genes Cells 14, 1067-1077. doi:10.1111/j.1365-2443.2009.01335.x

Kazazic, M., Roepstorff, K., Johannessen, L. E., Pedersen, N. M., van Deurs, B., Stang, E., et al. (2006). EGF-induced Activation of the EGF Receptor Does Not Trigger Mobilization of Caveolae. Traffic 7, 1518-1527. doi:10.1111/j.16000854.2006.00487.x

Kim, H., He, Y., Yang, I., Zeng, Y., Kim, Y., Seo, Y.-W., et al. (2012). $\delta$-Catenin Promotes E-Cadherin Processing and Activates $\beta$-catenin-mediated Signaling: Implications on Human Prostate Cancer Progression. Biochim. Biophys. Acta (Bba) - Mol. Basis Dis. 1822, 509-521. doi:10.1016/j.bbadis.2011.12.015

Kim, M. H., Rebbert, M. L., Ro, H., Won, M., and Dawid, I. B. (2014). Cell Adhesion in Zebrafish Embryos Is Modulated by March8. PLOS ONE 9, e94873. doi:10.1371/journal.pone.0094873

Kon, S., Tanabe, K., Watanabe, T., Sabe, H., and Satake, M. (2008). Clathrin Dependent Endocytosis of E-Cadherin Is Regulated by the Arf6GAP Isoform SMAP1. Exp. Cel Res. 314, 1415-1428. doi:10.1016/j.yexcr.2007.11.006

Kourtidis, A., Ngok, S. P., and Anastasiadis, P. Z. (2013). p120 Catenin. Prog. Mol. Biol. Transl Sci. 116, 409-432. doi:10.1016/B978-0-12-394311-8.00018-2

Kourtidis, A., Yanagisawa, M., Huveldt, D., Copland, J. A., and Anastasiadis, P. Z. (2015). Pro-Tumorigenic Phosphorylation of P120 Catenin in Renal and Breast Cancer. PLOS ONE 10, e0129964. doi:10.1371/journal.pone.0129964

Kowalczyk, A. P., and Nanes, B. A. (2012). Adherens junction Turnover: Regulating Adhesion through Cadherin Endocytosis, Degradation, and Recycling. Subcell Biochem. 60, 197-222. doi:10.1007/978-94-007-4186-7_9

Lambert, S., Vind-Kezunovic, D., Karvinen, S., and Gniadecki, R. (2006). LigandIndependent Activation of the EGFR by Lipid Raft Disruption. J. Invest. Dermatol. 126, 954-962. doi:10.1038/sj.jid. 5700168

Le, P. U., Guay, G., Altschuler, Y., and Nabi, I. R. (2002). Caveolin-1 Is a Negative Regulator of Caveolae-Mediated Endocytosis to the Endoplasmic Reticulum. J. Biol. Chem. 277, 3371-3379. doi:10.1074/jbc.M111240200

Le, T. L., Yap, A. S., and Stow, J. L. (1999). Recycling of E-Cadherin. J. Cel Biol 146, 219-232. doi:10.1083/jcb.146.1.219

Lee, D. M., and Harris, T. J. C. (2013). An Arf-GEF Regulates Antagonism between Endocytosis and the Cytoskeleton for Drosophila Blastoderm Development. Curr. Biol. 23, 2110-2120. doi:10.1016/j.cub.2013.08.058

Levkowitz, G., Waterman, H., Ettenberg, S. A., Katz, M., Tsygankov, A. Y., Alroy, I., et al. (1999). Ubiquitin Ligase Activity and Tyrosine Phosphorylation Underlie Suppression of Growth Factor Signaling by C-Cbl/Sli-1. Mol. Cel 4, 1029-1040. doi:10.1016/s1097-2765(00)80231-2

Li, H., Qiu, Z., Li, F., and Wang, C. (2017). The Relationship between MMP-2 and MMP-9 Expression Levels with Breast Cancer Incidence and Prognosis. Oncol. Lett. 14, 5865-5870. doi:10.3892/ol.2017.6924

Lickert, H., Bauer, A., Kemler, R., and Stappert, J. (2000). Casein Kinase II Phosphorylation of E-Cadherin Increases E-Cadherin/ $\beta$-Catenin Interaction and Strengthens Cell-Cell Adhesion. J. Biol. Chem. 275, 5090-5095. doi:10.1074/jbc.275.7.5090

Liu, Z., Zanata, S. M., Kim, J., Peterson, M. A., Di Vizio, D., Chirieac, L. R., et al. (2013). The Ubiquitin-specific Protease USP2a Prevents Endocytosis-Mediated EGFR Degradation. Oncogene 32, 1660-1669. doi:10.1038/onc.2012.188

Lu, Z., Ghosh, S., Wang, Z., and Hunter, T. (2003). Downregulation of Caveolin-1 Function by EGF Leads to the Loss of E-Cadherin, Increased Transcriptional Activity of $\beta$-catenin, and Enhanced Tumor Cell Invasion. Cancer Cell 4, 499-515. doi:10.1016/S1535-6108(03)00304-0

Mariner, D. J., Davis, M. A., and Reynolds, A. B. (2004). EGFR Signaling to P120Catenin through Phosphorylation at Y228. J. Cel Sci 117, 1339-1350. doi:10.1242/jcs.01001

Marmor, M. D., and Yarden, Y. (2004). Role of Protein Ubiquitylation in Regulating Endocytosis of Receptor Tyrosine Kinases. Oncogene 23, 2057-2070. doi:10.1038/sj.onc.1207390

McEwen, A. E., Maher, M. T., Mo, R., and Gottardi, C. J. (2014). E-cadherin Phosphorylation Occurs during its Biosynthesis to Promote its Cell Surface Stability and Adhesion. MBoC 25, 2365-2374. doi:10.1091/mbc.E14-01-0690

Miloso, M., Mazzotti, M., Vass, W. C., and Beguinot, L. (1995). SHC and GRB-2 Are Constitutively Activated by an Epidermal Growth Factor Receptor with a
Point Mutation in the Transmembrane Domain. J. Biol. Chem. 270, 19557-19562. doi:10.1074/jbc.270.33.19557

Mohan, A., Schlue, K. T., Kniffin, A. F., Mayer, C. R., Duke, A. A., Narayanan, V., et al. (2018). Spatial Proliferation of Epithelial Cells Is Regulated by E-Cadherin Force. Biophysical J. 115, 853-864. doi:10.1016/j.bpj.2018.07.030

Molès, J.-P., and Watt, F. M. (1997). The Epidermal Stem Cell Compartment: Variation in Expression Levels of E-Cadherin and Catenins within the Basal Layer of Human Epidermis. J. Histochem. Cytochem. 45, 867-874. doi:10.1177/ 002215549704500611

Navarini, N. F., De Araújo, V. C., Sperandio, M., Napimoga, M. H., Teixeira, L. N., De Araújo, N. S., et al. (2017). Effect of Epithelial Growth Factor on Matrix Metalloproteinase-2 and E-Cadherin/ $\beta$-Catenin Expression in an In Situ Model of Tumorigenesis. Oncol. Lett. 14, 3136-3140. doi:10.3892/ol.2017.6513

Niedzwiecki, M. W., Monterrey, J. C., Kalinovsky, T., Rath, M., and Niedzwiecki, A. (2009). Patterns of MMP-2 and MMP-9 Expression in Human Cancer Cell Lines. Oncol. Rep. 21, 1323-1333. doi:10.3892/or_00000358

O’Keefe, D. D., Prober, D. A., Moyle, P. S., Rickoll, W. L., and Edgar, B. A. (2007). Egfr/Ras Signaling Regulates DE-cadherin/Shotgun Localization to Control Vein Morphogenesis in the Drosophila wing. Developmental Biol. 311, 25-39. doi:10.1016/j.ydbio.2007.08.003

Oda, K., Matsuoka, Y., Funahashi, A., and Kitano, H. (2005). A Comprehensive Pathway Map of Epidermal Growth Factor Receptor Signaling. Mol. Syst. Biol. 1, 2005.0010. doi:10.1038/msb4100014

Oldrini, B., Hsieh, W.-Y., Erdjument-Bromage, H., Codega, P., Carro, M. S., CurielGarcía, A., et al. (2017). EGFR Feedback-Inhibition by Ran-Binding Protein 6 Is Disrupted in Cancer. Nat. Commun. 8, 2035. doi:10.1038/s41467-017-02185-w

Orlichenko, L., Huang, B., Krueger, E., and McNiven, M. A. (2006). Epithelial Growth Factor-Induced Phosphorylation of Caveolin 1 at Tyrosine 14 Stimulates Caveolae Formation in Epithelial Cells. J. Biol. Chem. 281, 4570-4579. doi:10.1074/jbc.M512088200

Palacios, F., Tushir, J. S., Fujita, Y., and D'Souza-Schorey, C. (2005). Lysosomal Targeting of E-Cadherin: a Unique Mechanism for the Down-Regulation of Cell-Cell Adhesion during Epithelial to Mesenchymal Transitions. Mol. Cel Biol 25, 389-402. doi:10.1128/MCB.25.1.389-402.2005

Pareja, F., Ferraro, D. A., Rubin, C., Cohen-Dvashi, H., Zhang, F., Aulmann, S., et al. (2012). Deubiquitination of EGFR by Cezanne-1 Contributes to Cancer Progression. Oncogene 31, 4599-4608. doi:10.1038/onc.2011.587

Parsons, J. T., Horwitz, A. R., and Schwartz, M. A. (2010). Cell Adhesion: Integrating Cytoskeletal Dynamics and Cellular Tension. Nat. Rev. Mol. Cel Biol 11, 633-643. doi:10.1038/nrm2957

Pece, S., and Gutkind, J. S. (2002). E-cadherin and Hakai: Signalling, Remodeling or Destruction? Nat. Cel Biol 4, E72-E74. doi:10.1038/ncb0402-e72

Pece, S., and Gutkind, J. S. (2000). Signaling from E-Cadherins to the MAPK Pathway by the Recruitment and Activation of Epidermal Growth Factor Receptors upon Cell-Cell Contact Formation. J. Biol. Chem. 275, 41227-41233. doi:10.1074/jbc.M006578200

Piedra, J., Miravet, S., Castaño, J., Pa'Imer, H. G., Heisterkamp, N., Garci'a de Herreros, A., et al. (2003). p120 Catenin-Associated Fer and Fyn Tyrosine Kinases Regulate $\beta$-Catenin Tyr-142 Phosphorylation and $\beta$-Catenin- $\alpha$-Catenin Interaction. Mol. Cel Biol 23, 2287-2297. doi:10.1128/MCB.23.7.2287-2297.2003

Pinilla-Macua, I., Grassart, A., Duvvuri, U., Watkins, S. C., and Sorkin, A. (2017). EGF Receptor Signaling, Phosphorylation, Ubiquitylation and Endocytosis in Tumors In Vivo. eLife 6, e31993. doi:10.7554/eLife.31993

Pol, A., Calvo, M., Lu, A., and Enrich, C. (2000). EGF Triggers Caveolin Redistribution from the Plasma Membrane to the Early/sorting Endocytic Compartment of Hepatocytes. Cell Signal. 12, 537-540. doi:10.1016/s08986568(00)00100-5

Porębska, N., Poźniak, M., Matynia, A., Żukowska, D., Zakrzewska, M., Otlewski, J., et al. (2021). Galectins as Modulators of Receptor Tyrosine Kinases Signaling in Health and Disease. Cytokine Growth Factor. Rev. 60, 89-106. doi:10.1016/ j.cytogfr.2021.03.004

Proux-Gillardeaux, V., Advedissian, T., Perin, C., Gelly, J.-C., Viguier, M., and Deshayes, F. (2021). Identification of a New Regulation Pathway of EGFR and E-Cadherin Dynamics. Sci. Rep. 11, 22705. doi:10.1038/s41598-021-02042-3

Qian, X., Karpova, T., Sheppard, A. M., McNally, J., and Lowy, D. R. (2004). E-cadherin-mediated Adhesion Inhibits Ligand-dependent Activation of Diverse Receptor Tyrosine Kinases. EMBO J. 23, 1739-1784. doi:10.1038/ sj.emboj.7600136 
Ramirez Moreno, M., Stempor, P. A., and Bulgakova, N. A. (2021). Interactions and Feedbacks in E-Cadherin Transcriptional Regulation. Front. Cel Dev. Biol. 9. doi:10.3389/fcell.2021.701175

Rappoport, J. Z., and Simon, S. M. (2009). Endocytic Trafficking of Activated EGFR Is AP-2 Dependent and Occurs through Preformed Clathrin Spots. J. Cel Sci 122, 1301-1305. doi:10.1242/jcs.040030

Reynolds, A. B., and Roczniak-Ferguson, A. (2004). Emerging Roles for P120Catenin in Cell Adhesion and Cancer. Oncogene 23, 7947-7956. doi:10.1038/ sj.onc. 1208161

Roura, S., Miravet, S., Piedra, J., de Herreros, A. G., and Duñach, M. (1999). Regulation of E-cadherin/Catenin Association by Tyrosine Phosphorylation. J. Biol. Chem. 274, 36734-36740. doi:10.1074/jbc.274.51.36734

Rowinsky, E. K. (2004). The erbB Family: Targets for Therapeutic Development against Cancer and Therapeutic Strategies Using Monoclonal Antibodies and Tyrosine Kinase Inhibitors. Annu. Rev. Med. 55, 433-457. doi:10.1146/ annurev.med.55.091902.104433

Rübsam, M., Mertz, A. F., Kubo, A., Marg, S., Jüngst, C., Goranci-Buzhala, G., et al. (2017). E-cadherin Integrates Mechanotransduction and EGFR Signaling to Control Junctional Tissue Polarization and Tight junction Positioning. Nat. Commun. 8, 1250. doi:10.1038/s41467-017-01170-7

Sato, K., Watanabe, T., Wang, S., Kakeno, M., Matsuzawa, K., Matsui, T., et al. (2011). Numb Controls E-Cadherin Endocytosis through P120 Catenin with aPKC. $M B \circ C 22,3103-3119$. doi:10.1091/mbc.E11-03-0274

Schnitzer, J. E., Oh, P., Pinney, E., and Allard, J. (1994). Filipin-sensitive CaveolaeMediated Transport in Endothelium: Reduced Transcytosis, Scavenger Endocytosis, and Capillary Permeability of Select Macromolecules. J. Cel Biol 127, 1217-1232. doi:10.1083/jcb.127.5.1217

Sehgal, P., Kong, X., Wu, J., Sunyer, R., Trepat, X., and Leckband, D. (2018). Epidermal Growth Factor Receptor and Integrins Control Force-dependent Vinculin Recruitment to E-Cadherin Junctions. J. Cel Sci 131, jcs206656. doi:10.1242/jcs.206656

Shrestha, H., Ryu, T., Seo, Y.-W., Park, S.-Y., He, Y., Dai, W., et al. (2017). Hakai, an E3-Ligase for E-Cadherin, Stabilizes $\delta$-catenin through Src Kinase. Cell Signal. 31, 135-145. doi:10.1016/j.cellsig.2017.01.009

Sigismund, S., Algisi, V., Nappo, G., Conte, A., Pascolutti, R., Cuomo, A., et al. (2013). Threshold-controlled Ubiquitination of the EGFR Directs Receptor Fate. Embo J. 32, 2140-2157. doi:10.1038/emboj.2013.149

Sigismund, S., Argenzio, E., Tosoni, D., Cavallaro, E., Polo, S., and Di Fiore, P. P. (2008). Clathrin-Mediated Internalization Is Essential for Sustained EGFR Signaling but Dispensable for Degradation. Developmental Cel 15, 209-219. doi:10.1016/j.devcel.2008.06.012

Sigismund, S., Avanzato, D., and Lanzetti, L. (2018). Emerging Functions of the EGFR in Cancer. Mol. Oncol. 12, 3-20. doi:10.1002/1878-0261.12155

Sigismund, S., Woelk, T., Puri, C., Maspero, E., Tacchetti, C., Transidico, P., et al. (2005). Clathrin-independent Endocytosis of Ubiquitinated Cargos. Proc. Natl. Acad. Sci. 102, 2760-2765. doi:10.1073/pnas.0409817102

Simón, L., Campos, A., Leyton, L., and Quest, A. F. G. (2020). Caveolin-1 Function at the Plasma Membrane and in Intracellular Compartments in Cancer. Cancer Metastasis Rev. 39, 435-453. doi:10.1007/s10555-020-09890-x

Singh, B., Carpenter, G., and Coffey, R. J. (2016). EGF Receptor Ligands: Recent Advances. F1000Res 5, 2270. doi:10.12688/f1000research.9025.1

Song, H., Huang, L., Zhang, M., Wang, X., Song, S., and Yang, L. (2014). Transphosphorylation of EGFR at Y845 Plays an Important Role in its Autophosphorylation and Kinase Activity. Oncol. Rep. 31, 2393-2398. doi:10.3892/or.2014.3102

Takahashi, K., and Suzuki, K. (1996). Density-dependent Inhibition of Growth Involves Prevention of EGF Receptor Activation by E-Cadherin-Mediated CellCell Adhesion. Exp. Cel Res. 226, 214-222. doi:10.1006/excr.1996.0221

Takeichi, M. (1977). Functional Correlation between Cell Adhesive Properties and Some Cell Surface Proteins. J. Cel Biol. 75, 464-474. doi:10.1083/jcb.75.2.464

Thelemann, A., Petti, F., Griffin, G., Iwata, K., Hunt, T., Settinari, T., et al. (2005). Phosphotyrosine Signaling Networks in Epidermal Growth Factor Receptor Overexpressing Squamous Carcinoma Cells. Mol. Cell Proteomics 4, 356-376. doi:10.1074/mcp.M400118-MCP200

Thien, C. B. F., and Langdon, W. Y. (2005). Mini ReviewNegative Regulation of PTK Signalling by Cbl Proteins. Growth Factors 23, 161-167. doi:10.1080/ 08977190500153763
Tong, J., Taylor, P., Peterman, S. M., Prakash, A., and Moran, M. F. (2009). Epidermal Growth Factor Receptor Phosphorylation Sites Ser991 and Tyr998 Are Implicated in the Regulation of Receptor Endocytosis and Phosphorylations at Ser1039 and Thr1041. Mol. Cell Proteomics 8, 2131-2144. doi:10.1074/mcp.M900148-MCP200

Vecchione, L., Jacobs, B., Normanno, N., Ciardiello, F., and Tejpar, S. (2011). EGFR-targeted Therapy. Exp. Cel Res. 317, 2765-2771. doi:10.1016/ j.yexcr.2011.08.021

Wang, D., Su, L., Huang, D., Zhang, H., Shin, D. M., and Chen, Z. G. (2011). Downregulation of E-Cadherin Enhances Proliferation of Head and Neck Cancer through Transcriptional Regulation of EGFR. Mol. Cancer 10, 116. doi:10.1186/1476-4598-10-116

Wang, Y., Hu, J., Wang, Y. a., Ye, W., Zhang, X., Ju, H., et al. (2018). EGFR Activation Induced Snail-dependent EMT and Myc-dependent PD-L1 in Human Salivary Adenoid Cystic Carcinoma Cells. Cell Cycle 17, 1457-1470. doi:10.1080/15384101.2018.1489177

Wee, P., and Wang, Z. (2017). Epidermal Growth Factor Receptor Cell Proliferation Signaling Pathways. Cancers 9, 52. doi:10.3390/cancers 9050052

Xiao, K., Allison, D. F., Kottke, M. D., Summers, S., Sorescu, G. P., Faundez, V., et al. (2003). Mechanisms of VE-Cadherin Processing and Degradation in Microvascular Endothelial Cells. J. Biol. Chem. 278, 19199-19208. doi:10.1074/ jbc.M211746200

Yanagisawa, M., Huveldt, D., Kreinest, P., Lohse, C. M., Cheville, J. C., Parker, A. S., et al. (2008). A P120 Catenin Isoform Switch Affects Rho Activity, Induces Tumor Cell Invasion, and Predicts Metastatic Disease. J. Biol. Chem. 283, 18344-18354. doi:10.1074/jbc.M801192200

Yang, W.-N., Ai, Z.-H., Wang, J., Xu, Y.-L., and Teng, Y.-C. (2014). Correlation between the Overexpression of Epidermal Growth Factor Receptor and Mesenchymal Makers in Endometrial Carcinoma. J. Gynecol. Oncol. 25, 36-42. doi:10.3802/jgo.2014.25.1.36

Yarden, Y. (2001). The EGFR Family and its Ligands in Human Cancer. Signalling Mechanisms and Therapeutic Opportunities. Eur. J. Cancer 37 (Suppl. 4), S3-S8. doi:10.1016/s0959-8049(01)00230-1

Yu, W., Yang, L., Li, T., and Zhang, Y. (2019). Cadherin Signaling in Cancer: Its Functions and Role as a Therapeutic Target. Front. Oncol. 9, 989. doi:10.3389/ fonc.2019.00989

Zhao, H., Liang, Y., Xu, Z., Wang, L., Zhou, F., Li, Z., et al. (2008). N-glycosylation Affects the Adhesive Function of E-Cadherin through Modifying the Composition of Adherens Junctions (AJs) in Human Breast Carcinoma Cell Line MDA-MB-435. J. Cell. Biochem. 104, 162-175. doi:10.1002/jcb.21608

Zhitnyak, I. Y., Rubtsova, S. N., Litovka, N. I., and Gloushankova, N. A. (2020). Early Events in Actin Cytoskeleton Dynamics and E-Cadherin-Mediated CellCell Adhesion during Epithelial-Mesenchymal Transition. Cells 9, 578. doi: $10.3390 /$ cells 9030578

Zuo, J.-H., Zhu, W., Li, M.-Y., Li, X.-H., Yi, H., Zeng, G.-Q., et al. (2011). Activation of EGFR Promotes Squamous Carcinoma SCC10A Cell Migration and Invasion via Inducing EMT-like Phenotype Change and MMP-9-Mediated Degradation of E-Cadherin. J. Cell. Biochem. 112, 2508-2517. doi:10.1002/jcb.23175

Conflict of Interest: The authors declare that the research was conducted in the absence of any commercial or financial relationships that could be construed as a potential conflict of interest.

Publisher's Note: All claims expressed in this article are solely those of the authors and do not necessarily represent those of their affiliated organizations, or those of the publisher, the editors and the reviewers. Any product that may be evaluated in this article, or claim that may be made by its manufacturer, is not guaranteed or endorsed by the publisher.

Copyright (c) 2022 Ramírez Moreno and Bulgakova. This is an open-access article distributed under the terms of the Creative Commons Attribution License (CC BY). The use, distribution or reproduction in other forums is permitted, provided the original author(s) and the copyright owner(s) are credited and that the original publication in this journal is cited, in accordance with accepted academic practice. No use, distribution or reproduction is permitted which does not comply with these terms. 